EQUAÇÕES PARA ESTIMAR ÁREA FOLIAR DE MARACUJÁ AMARELO

SCHMILDT, Edilson Romais ${ }^{1}$

NEGRIS, Fernanda Segantini ${ }^{2}$

CESANA JÚNIOR, Eduardo ${ }^{2}$

SCHMILDT, Omar ${ }^{2}$

ALEXANDRE, Rodrigo Sobreira ${ }^{3}$

RESUMO: O conhecimento da área foliar é fundamental, por ser importante em diversas áreas, principalmente na fisiologia e na avaliação do desenvolvimento de plantas. O objetivo deste trabalho foi avaliar diferentes modelos estatísticos e identificar o que melhor se ajusta à área foliar de maracujá amarelo (Passiflora edulis Sims. f. flavicarpa Degener) a partir de medidas lineares do limbo foliar. Foram coletadas 192 folhas aleatoriamente de 20 plantas, e, realizadas as medições de comprimento do lóbulo central (CLC), maior largura do lóbulo central (LLC), maior largura da folha (LF) e, área foliar real (AFR), sendo esta por meio de imagens digitais. Foram avaliados os modelos linear, circunscrito, potencial e exponencial, usando a AFR em função de CLC, LLC, LF, CLCxLF e CLCxLLC. O ajuste mais adequado exige o uso das medidas CLC e LLC, sendo que a equação indicada é $\mathrm{AF}=1,5885$ (CLCXLLC).

Palavras-chave: Passiflora edulis Sims. f. flavicarpa Degener. Método não destrutivo. Modelagem estatística.

\title{
EQUATIONS FOR DETERMINATION OF LEAF AREA OF YELLOW PASSION FRUIT
}

SUMMARY: Knowledge of the leaf area is critical to be important in several areas, especially in physiology, to evaluate the development of plants. The objective of this study was to evaluate different statistical models and identify what best fits leaf area yellow passion fruit (Passiflora edulis Sims. f. flavicarpa Degener) from linear measurements of the leaf.Were collected randomly 192 leaves from of 20 plants, and the measurements of length of the central lobe (LCL), the greater width of the central lobe (WCL), the greater width of the leaf (WF) and real leaf area (RLA), which is by means of digital images. Were evaluated the linear models, circumscribed, potential and exponential, using the RLAin function of LCL, WCL, WF, LCLxWCLand LCLxWCL. The most appropriate setting requires the use of LCL measures and WCL, and the equation is indicated is LA $=1.5885$ (LCLCxWCL).

Keywords: Passiflora edulis Sims. f. flavicarpa Degener. Non-destructive method. Statistic model.

\section{INTRODUÇÃO}

O Brasil é um dos maiores produtores mundiais de maracujá, sendo que no ano de 2013 produziu o equivalente a 838.244 toneladas da fruta em área colhida de 57.277 hectares (IBGE, 2014). O bom desempenho do Brasil na produção de maracujá é resultado do trabalho em diversas áreas, dentre outras a fitotécnica (ANDRADE JÚNIOR et al., 2003; NEGREIROS et al., 2007) e o melhoramento (MORGADO et al., 2010; FALEIRO et al., 2011), principalmente referentes ao maracujá amarelo (P. edulis). Além da produção de suco, esta espécie tem sido usada na América do Sul como sedativa, diurética, antihelmíntica, antidiarreica, estimulante, tônica, no tratamento da hipertensão e para os sintomas da menopausa e cólica

\footnotetext{
${ }^{1}$ UFES - Departamento de Ciências Agrárias e Biológicas (DCAB); Genética e Melhoramento de Plantas.

${ }^{2}$ UFES - Produção vegetal

${ }^{3}$ UFES - Departamento de Ciências Florestais e da Madeira
} 
infantil (DHAWAN et al., 2004).

Independente de qual seja a área de pesquisa, na maioria das vezes, em trabalhos de campo, é necessário o conhecimento da área foliar da cultura ou da folha individualmente (AMARAL et al., 2009). Assim, a área foliar é importante para determinar o volume de calda de defensivos (FERREIRA et al., 2010), em avaliações fisiológicas (AMARAL et al., 2009), dentre outras. Diante desta constatação, Morgado et al. (2013) trabalharam na determinação de área foliar por método não destrutivo em oito espécies do gênero Passiflora, porém dentre estas não inclui $P$. edulis.

Os modelos estatísticos que relacionam as medidas lineares da folha e sua área real para estimar a área foliar (BENINCASA, 2003; AMARAL et al., 2009) são métodos não destrutivos, de boa precisão e baixo custo, eliminando a necessidade de medidores de área foliar caros ou a realização de demoradas reconstruções geométricas (GAMIELY et al., 1991).

O estudo da modelagem da área foliar a partir de medidas lineares do limbo foliar tem sido usado para frutíferas como pinha (SILVA et al., 2004), laranja (MAZZINI et al., 2010), mangueira (LIMA et al., 2012) e abacaxi (FRANCISCO et al., 2014).

O presente trabalho teve como objetivo avaliar modelos estatísticos para estimar a área foliar, por método não destrutivo, da espécie do maracujá amarelo ( $P$. edulis) e indicar uma equação de regressão que melhor represente a área foliar em função de medidas alométricas das folhas.

\section{MATERIAL E MÉTODO}

Neste trabalho foram utilizadas 192 folhas, coletadas em todos os estágios de desenvolvimento, de 20 plantas de maracujá amarelo ( $P$. edulis), conforme sugerido por Benincasa (2003), obtidas no assentamento Vale da Vitória, BR 381, São Mateus, Espírito Santo, no mês de fevereiro de 2013. As folhas foram coletadas, sem danos, e acondicionadas em sacos plásticos, transferidas para o Laboratório de Genética e Melhoramento de Plantas no CEUNES/UFES. Em laboratório foram determinadas as dimensões lineares da folha, com a utilização de uma régua milimetrada, em que: CLC $=$ comprimento do lóbulo central; LLC = largura maior do lóbulo central; LF = largura da folha. As medidas de CLC, LLC e LF são ilustradas na Figura 1. A partir das dimensões lineares das folhas calculou-se também os produtos CLCXLLC e CLCXLF.

Figura 1. Representação gráfica do comprimento do lóbulo central (CLC), maior largura do lóbulo central (LLC) e maior largura da folha (LF) de uma folha do maracujazeiro amarelo (P. edulis)

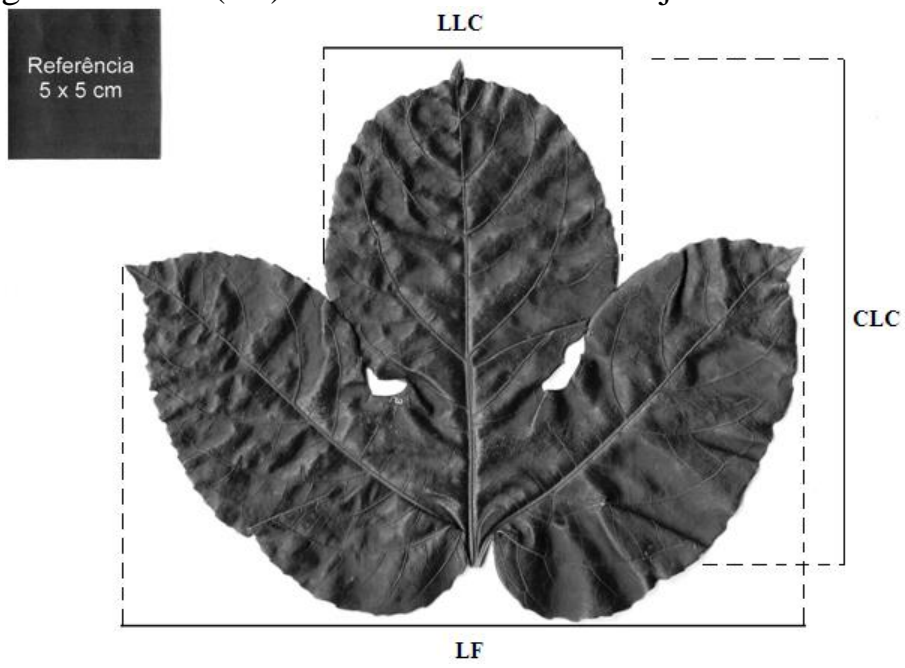

Fonte: Elaborado pelos autores 
A área foliar real (AFR) foi determinada a partir de imagens digitais das folhas, feitas com escâner HP Deskjet F4480 ${ }^{\circledR}$, em formato tiff com 75 dpi e, processadas no software ImageJ ${ }^{\circledR}$ (COLLINS, 2007). Este software, que é de domínio público, foi usado com sucesso na determinação da área foliar de batata Agata (JADOSKI et al., 2012), feijão (MARTIN et al., 2013) e de cacaueiro (SANTOS et al., 2014).

Para caracterizar o CLC, LLC, LF, CLCxLLC, CLCxLF e AFR das folhas, foram feitas análises da estatística descritiva e do tamanho mínimo da amostra requerido para o estudo de modelagem. O número de folhas para estimar os parâmetros das medidas foliares em uma população infinita de maracujá com uma confiança de 95\% foi calculada pela seguinte equação, descrita por Cochran (1977):

$\eta=\frac{\left(t_{\alpha / 2} s\right)^{2}}{(e m)^{2}}$

Onde: $\eta$ é o tamanho da amostra (número de folhas de maracujá); $t_{\alpha / 2}$ é o valor crítico da distribuição t de Student, cuja área à direita é igual a $\alpha / 2$ com $(n-1)$ graus de liberdade, e com $5 \%$ de probabilidade de erro; s é o desvio padrão amostral; e é o erro na estimativa da média, fixado em 0,05 ; $\mathrm{m}$ é a média amostral.

Para modelar a área foliar observada (variável dependente $=Y_{i}$ ) em função do CLC, da LLC, da LF, do CLCxLLC e, do CLCxLF, como variáveis independentes $\left(\mathrm{x}_{\mathrm{i}}\right)$, utilizou-se os modelos linear $\left(\hat{\mathrm{Y}}_{\mathrm{i}}=\hat{\beta}_{0}+\hat{\beta}_{1} \mathrm{x}_{\mathrm{i}}\right)$, circunscrito $\left(\hat{\mathrm{Y}}_{\mathrm{i}}=\hat{\beta}_{1} \mathrm{x}_{\mathrm{i}}\right)$, potência $\left(\hat{\mathrm{Y}}_{\mathrm{i}}=\hat{\beta}_{0} \mathrm{x}_{\mathrm{i}} \hat{\beta}_{1}\right)$ e, exponencial $\left(\hat{\mathrm{Y}}_{\mathrm{i}}=\hat{\beta}_{0} \hat{\beta}_{1}^{\mathrm{X}}\right)$. Os parâmetros $\beta_{0}$ e $\beta_{1}$ foram estimados pelo método dos mínimos quadrados tendo sido feita previamente a linearização das funções potência e exponencial.

Para realizar as comparações entre os modelos, foram obtidas as somas de quadrados das diferenças entre os valores observados e os preditos pelos modelos, o que foi denominado de soma de quadrados do desvio. No caso dos modelos com transformação (potência e exponencial), foi feito o retorno para a escala original e, após isso, obtidas as referidas somas de quadrados do resíduo. Os coeficientes de determinação nestes modelos foram obtidos como o quadrado da correlação entre os valores observados e estimados na escala original (SOUZA, 1998). A melhor equação foi a que apresentou a menor soma de quadrados do desvio na escala real e o maior coeficiente de determinação. As análises estatísticas foram realizadas com auxílio dos programas Genes (CRUZ, 2013) e R (R DEVELOPMENT CORE TEAM, 2014).

\section{RESULTADO E DISCUSSÃO}

Na Tabela 1 estão descritos os dados de medidas de posição e de variabilidade para comprimento do lóbulo central (CLC), largura maior do lóbulo central (LLC), largura da folha (LF) e área foliar real (AFR) das folhas de P. edulis. Na amostra de 192 folhas houve algumas com valores de CLC e LF inferiores e outras superiores aos valores citados para a espécie por Beraldo e Kato (2010). Esta amplitude nas medidas lineares das folhas é traduzida em dispersão em torno da média, com coeficientes de variação considerados médios para CLC, LLC e LF e muito altos para CLCxLLC, CLCxLF e AFR (PIMENTELGOMES, 2009). Estes resultados apontam que o estudo de modelagem pode ser executado para aplicação a folhas de maracujazeiro amarelo de diversos tamanhos. A maior variabilidade da AFR em relação às dimensões lineares de comprimento e de largura segue a tendência observada na modelagem de área foliar em outras culturas agrícolas como mangueira (LIMA et al., 2012) e feijão guandu (CARGNELUTTI FILHO et al., 2015). 
Tabela 1. Média, mínimo, máximo, desvio padrão (DP), coeficiente de variação (CV\%) e, número mínimo de folhas requeridas (n) para o estudo de área foliar do maracujazeiro amarelo ( $P$. edulis) a partir de suas dimensões lineares do comprimento do lóbulo central (CLC), da maior largura do lóbulo central (LLC), maior largura da folha (LF), produto entre CLCxLLC, produto entre CLCxLF e de sua área foliar real (AFR) medidas em 192 folhas

\begin{tabular}{ccccccc}
\hline Caracteres & Médias & Mínimos & Máximos & DP & CV\% & $\mathrm{n}^{(1)}$ \\
\hline CLC & 12,53 & 6,20 & 17,50 & 2,00 & 16,00 & 10 \\
LLC & 6,49 & 0,70 & 9,60 & 1,26 & 19,39 & 15 \\
LF & 17,44 & 4,10 & 27,30 & 3,45 & 19,76 & 16 \\
CLCxLLC & 83,68 & 20,46 & 162,75 & 27,29 & 32,61 & 42 \\
CLCxLF & 224,55 & 60,83 & 477,75 & 74,07 & 32,98 & 43 \\
AFR & 132,29 & 33,64 & 274,83 & 46,94 & 35,48 & 49 \\
\hline
\end{tabular}

(1) tamanho da amostra para uma confiança de 95\% calculado segundo Cochran (1977).

Os resultados referentes ao tamanho da amostra apontam que são necessários 49 ou mais folhas para o estudo da área foliar do maracujazeiro amarelo (Tabela 1). Portanto, o tamanho amostral usado neste trabalho, de 192 folhas, confere credibilidade para o referido estudo.

Quanto à modelagem da área foliar, na prática, o uso de equações de modelos lineares baseados em apenas uma dimensão linear é preferível por sua simplicidade para aplicação, principalmente no campo (LU et al., 2004). No caso deste trabalho, no entanto, não se indica o uso de apenas uma medida linear, pois todas as equações ajustadas para CLC, LLC e LF tiveram elevados valores do quadrado médio do desvio da regressão e coeficientes de determinação $\left(\mathrm{R}^{2}\right)$ abaixo de $90 \%$ (Tabela 2). Dentre as três medidas lineares, a LF foi a menos apropriada para o ajuste. Resultados semelhantes foram observados por Borghezan et al. (2010) em videira, que também possui o limbo foliar constituído de lóbulos. Os referidos autores observam que, na prática, há maior dificuldade na execução da medida entre os lóbulos laterais, não indicando o seu uso em folhas de videira.

Tabela 2. Equações de regressão estimadas, graus de liberdade (GL), quadrado médio de desvios (QMDesvio) da regressão, coeficientes de determinação $\left(\mathrm{R}^{2}\right.$, em \%), da área foliar, em função das medidas lineares do limbo foliar do maracujazeiro amarelo (P. edulis)

(Continua)

\begin{tabular}{|c|c|c|c|c|c|}
\hline \multirow{2}{*}{$\mathbf{X}^{1 /}$} & \multirow{2}{*}{$\begin{array}{l}\text { Tipos de } \\
\text { equação }\end{array}$} & \multicolumn{2}{|c|}{ Desvio da regressão } & \multirow[b]{2}{*}{$\mathrm{R}^{2}$} & \multirow{2}{*}{ Equação estimada } \\
\hline & & GL & QMD & & \\
\hline CLC & Linear & 190 & 296,81 & 86,59 & $\mathrm{AF}=-140,7818+21,7981 \mathrm{X}$ \\
\hline LLC & Linear & 190 & 489,92 & 77,87 & $\mathrm{AF}=-81,3084+32,9244 \mathrm{X}$ \\
\hline $\mathbf{L F}$ & Linear & 190 & 605,10 & 72,67 & $\mathrm{AF}=-70,2137+11,6124 \mathrm{X}$ \\
\hline CLCxLLC & Linear & 190 & 152,83 & 93,09 & $A F=-6,6013+1,6598 X$ \\
\hline $\operatorname{CLCxLLC~}(0,0)$ & Circunscrito & 191 & 156,22 & 92,91 & $\mathrm{AF}=1,5885 \mathrm{X}$ \\
\hline CLCxLF & Linear & 190 & 233,02 & 89,47 & $A F=-2,3201+0,5994 X$ \\
\hline $\operatorname{CLCxLF}(0,0)$ & Circunscrito & 191 & 232,33 & 89,46 & $\mathrm{AF}=0,5902 \mathrm{X}$ \\
\hline
\end{tabular}


Tabela 2. Equações de regressão estimadas, graus de liberdade (GL), quadrado médio de desvios (QMDesvio) da regressão, coeficientes de determinação $\left(\mathrm{R}^{2}, \mathrm{em} \%\right)$, da área foliar, em função das medidas lineares do limbo foliar domaracujazeiro amarelo (P. edulis)

(Conclusão)

\begin{tabular}{|c|c|c|c|c|c|}
\hline \multirow{2}{*}{$\begin{array}{l}\mathbf{X}^{1 /} \\
\text { CLC }\end{array}$} & \multirow{2}{*}{$\begin{array}{l}\text { Tipos de } \\
\text { equação } \\
\text { Potência }\end{array}$} & \multicolumn{2}{|c|}{ Desvio da regressão } & \multirow[b]{2}{*}{88,85} & \multirow{2}{*}{$\begin{array}{l}\text { Equação estimada } \\
\mathrm{AF}=0,5671 \mathrm{X}^{2,1417}\end{array}$} \\
\hline & & 190 & 269,00 & & \\
\hline LLC & Potência & 190 & 707,02 & 51,21 & $\mathrm{AF}=16,1672 \mathrm{X}^{1,1025}$ \\
\hline $\mathbf{L F}$ & Potência & 190 & 620,37 & 63,04 & $\mathrm{AF}=2,6191 \mathrm{X}^{1,3591}$ \\
\hline CLC & Exponencial & 190 & 304,98 & 87,58 & $\mathrm{AF}=13,5368(1,1932)^{\mathrm{X}}$ \\
\hline LLC & Exponencial & 190 & 388,50 & 76,18 & $\mathrm{AF}=22,5566(1,3000)^{\mathrm{X}}$ \\
\hline $\mathbf{L F}$ & Exponencial & 190 & 557,46 & 71,33 & $\mathrm{AF}=24,5769(1,0971)^{\mathrm{X}}$ \\
\hline
\end{tabular}

${ }^{1}$ em que: CLC = comprimento do lóbulo central; LLC = largura maior do lóbulo central; LF = largura da folha

Figura 2. Representação gráfica da área foliar real em função do produto do comprimento do lóbulo central (CLC) pela largura máxima do lóbulo central (LLC) e equação linear que representa estatisticamente a relação em folhas do maracujazeiro amarelo ( $P$. edulis)

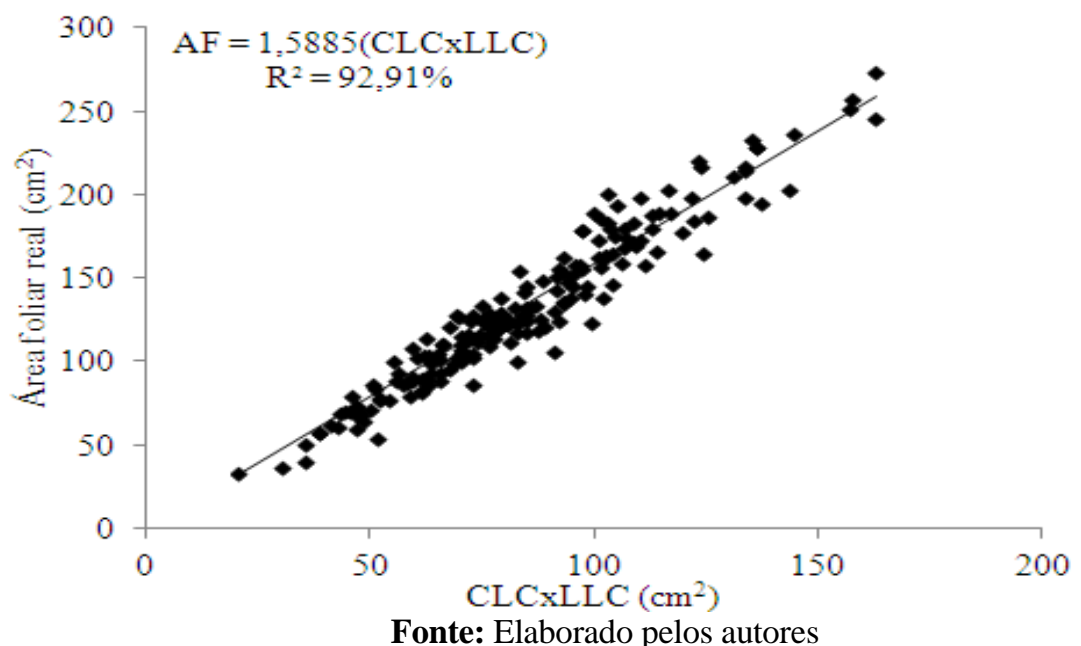

Os modelos estatísticos que mais se adequaram à área foliar do maracujazeiro amarelo foram o linear e o circunscrito que usaram o CLCxLLC como variável explicativa (Tabela 2). Por questões de simplicidade de cálculo, recomenda-se a equação AF =1,5885(CLCxLLC). Mazzini et al. (2010) em laranja e Francisco et al. (2014) em abacaxi, também concluíram que havia a necessidade do uso de medidas lineares de comprimento e de largura para melhor ajuste da área foliar por método não destrutivo.

\section{CONCLUSÃO}

A equação mais adequada para a estimativa de área foliar do maracujazeiro amarelo ( $P$. edulis) é $A F=1,5885(C L C x L L C)$, sendo CLC e LLC o comprimento e a largura do lóbulo central, respectivamente. 


\section{REFERÊNCIAS}

AMARAL, J.A.T. et al. Métodos de análise quantitativa do crescimento de plantas. In: FERREIRA, A. et al. (Eds). Tópicos especiais em produção vegetal I. Alegre: CCAUFES, 2009. p.259-276.

ANDRADE JUNIOR, V.C. et al. Produção de maracujazeiro amarelo sob diferentes densidades de plantio. Pesquisa Agropecuária Brasileira, Brasília, v.38, n.12, p.1381-1386, 2003. http://seer.sct.embrapa.br/index.php/pab/article/view/6715/3772

BENINCASA, M.M.P. Análise de crescimento de plantas: noções básicas. 2. ed. Jaboticabal: Funep, 2003. 41p.

BERALDO, J.; KATO, E.T.M. Morfoanatomia de folhas e caules de Passiflora edulis Sims, Passifloraceae. Revista Brasileira de Farmacognosia, Curitiba, v.20, n.2, p.233-239, 2010. http://www.scielo.br/pdf/rbfar/v20n2/a16v20n2.pdf

BORGHEZAN, M. et al. Modelos matemáticos para a estimativa da área foliar de variedades de videira à campo (Vitis vinifera L.). Ciência e Técnica Vitivinícola, Dois Portos, v.25, n.1, p.1-7, 2010. http://www.scielo.mec.pt/pdf/ctv/v25n1/v25n1a01.pdf

CARGNELUTTI FILHO, A. et al. Estimação da área foliar de feijão guandu por dimensões foliares.

Ciência Rural, Santa Maria, v.45, n.1, p.1-8, 2015.

http://www.scielo.br/scielo.php?pid=S0103-84782015000100001\&script=sci_arttext

COCHRAN, W.G. Sampling techniques. 3 ed. New York: John Wiley \& Sons, 1977. 428p.

COLLINS, T.J. ImageJ for microscopy. BioTechniques, Hamilton, v.43, n.1 supplement, p. 25-30, 2007. http://www.biotechniques.com/multimedia/archive/00003/BTN_A_000112517_O_3131a.pdf

CRUZ, C.D. GENES: a software package for analysis in experimental statistics and quantitative genetics. Acta Scientiarum. Agronomy. Maringá, v.35, n.3, p.271-276, 2013. http://periodicos.uem.br/ojs/index.php/ActaSciAgron/article/view/21251/pdf_1

DHAWAN, K.; DHAWAN, S.; SHARMA, A. Passiflora: a review update. Journal of Ethnopharmacology, Lausanne, v.94, n.1, p.1-23, 2004. http://www.sciencedirect.com/science/journal/03788741/94/1

FALEIRO, F.G. et al. Germoplasma e melhoramento genético do maracujazeiro: histórico e perspectivas. Planaltina: Embrapa Cerrados, 2011. 36 p. (Documentos/Embrapa Cerrados No 307).

FERREIRA, M.C. et al. Determinação de área foliar e retenção de líquido por folhas de café em pulverização a alto volume. Nucleus, Ituverava, v.7, n.1, p.277-283, 2010.

http://www.nucleus.feituverava.com.br/index.php/nucleus/article/view/316/407. Doi: 10.3738/1982.2278316.

FRANCISCO, J.P. et al. Estimativa da área foliar do abacaxizeiro cv. Vitória por meio de relações alométricas. Revista Brasileira de Fruticultura, Jaboticabal, v.36, n.2, p.285-293, 2014. http://www.readcube.com/articles/10.1590/0100-2945-216/13?tab=summary

GAMIELY, S. et al. A rapid and nondestructive method for estimating leaf area of onions. HortScience, Alexandria, v.26, n.2, p.206-206, 1991.

http://hortsci.ashspublications.org/content/26/2/206.short

IBGE. Banco de Dados Agregados: SIDRA. IBGE, 2014. Disponível em:

http://www.sidra.ibge.gov.br/bda/tabela/listabl.asp?c=1613\&z=p\&o=28 . Acesso em: 23 mar. 2015. 
JADOSKI, S. O. et al. Método de determinação da área foliar da cultivar de batata Ágata a partir de dimensões lineares. Semina: Ciências Agrárias, Londrina, v.33, suplemento 1, p. 2545-2554, 2012. http://www.uel.br/revistas/uel/index.php/semagrarias/article/viewFile/8058/11754

LIMA, R.T. et al. Modelos para estimativa da área foliar da mangueira utilizando medidas lineares. Revista Brasileira de Fruticultura, Jaboticabal, v.34, n.4, p.974-980, 2012. http://www.scielo.br/pdf/rbf/v34n4/03.pdf

LU, H.Y. et al. Comparison of different models for nondestructive leaf area estimation in taro. Agronomy Journal, Amsterdan, v.96, n.2, p.448-453, 2004.

https://www.agronomy.org/publications/aj/abstracts/96/2/448

MARTIN, T.N. et al. Uso do software ImageJ na estimativa de área foliar para a cultura do feijão. Interciencia, Caracas, v.38, n.12, p.843-848, 2013. http://www.interciencia.org/v38_12/843.pdf

MAZZINI, R.B.; RIBEIRO, R.V.; PIO, R.M. A simple and non-destructive model for individual leaf area estimation in Citrus. Fruits, Cambridge, v.65, n.5, p.269-275, 2010. http://journals.cambridge.org/download.php?file=\%2FFRU\%2FFRU65_05\%2FS0248129410000228a.pdf

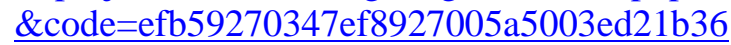

MORGADO, M.A.D.; SANTOS, C.E.M.; LINHARES, H.B,C.H. Correlações fenotípicas em características físico químicas do maracujazeiro-amarelo. Acta Agronômica, Bogotá, v.59, n.4, p.457461, 2010.

http://www.bdigital.unal.edu.co/23311/2/20130-67510-1-PB.pdf

MORGADO, M.A.D. et al. Estimação da área foliar por método não destrutivo, utilizando medidas lineares das folhas de espécies de Passiflora. Revista Ceres, Viçosa, v.60, n.5, p.662-667, 2013. http://www.scielo.br/pdf/rceres/v60n5/09.pdf

NEGREIROS, J.R.S et al. Relação entre características físicas e o rendimento de polpa de maracujáamarelo. Revista Brasileira de Fruticultura, Jaboticabal, v.29, n.3, p.546-549, 2007. http://www.scielo.br/scielo.php?script=sci arttext\&pid=S0100-29452007000300026

PIMENTEL-GOMES, F. Curso de estatística experimental. 15. ed. Piracicaba: Fealq, 2009. 451p.

R DEVELOPMENT CORE TEAM. R: A language and environment for statistical computing. $\mathbf{R}$ Foundation for Statistical Computing, Vienna. Disponível em: http://www.R-project.org/. Acesso em: 4 dez. 2014.

SANTOS, S.N et al. Análise comparativa de métodos de determinação de área foliar em genótipos de cacau. Bioscience Journal, Uberlandia, v. 30, supplement 1, p. 411-419, 2014.

http://www.seer.ufu.br/index.php/biosciencejournal/article/view/18148/14553

SILVA, P.S.L et al. Leaf area estimates of custard apple tree progenies. Revista Brasileira de Fruticultura, Jaboticabal, v.26, n.3, p.558-560, 2004.

http://www.scielo.br/scielo.php?script=sci arttext\&pid=S0100-29452004000300046

SOUZA, G.S. Introdução aos modelos de regressão linear e não-linear. Brasília: EMBRAPA, SPI/EMBRAPA-SEA, 1998. 505p. 
